\title{
Digital inclusion and gender-associated indicators: A critical review of post-2010 literature
}

\author{
Morten Meyerhoff Nielsen \\ United Nations University Operating Unit on \\ Policy-Driven Electronic Governance \\ meyerhoff@unu.edu
}

\author{
Mercy Erhi Makpor \\ United Nations University Operating Unit on \\ Policy-Driven Electronic Governance \\ makpor@unu.edu
}

\begin{abstract}
With the ever-increasing pace of technology innovation and rapid digital transformation of society, the impact on digital inclusion, and particularly gender equity is surprisingly under-analyzed. The article outlines and classifies the concept of digital inclusion and its effects on traditionally marginalized groups, specifically, girls and women. In doing so, the article reviews existing literature on digital inclusion and answers a core research question: What are some of the underlying monitoring indicators for monitoring and measuring the state of digital inclusion and digital gender divide identified in the academic literature to date? Based on a scoping review, an initial 8,527 documents published between 1951 and January 2021, were identified as having some relevance to the digital inclusion of marginalized communities. Our analysis found that 208 publications include gender. Of the 208 publications, 116 publications (56\%) were excluded as they focused mainly on technology use in healthcare provision and assisted living for adults and the elderly rather than digital inclusion and gender. The remaining 92 publications (44\%) covered gender relating to the digital inclusion of men and women, boys and girls, and the digital gender divide. Our review finds that the effects of digital inclusion on the traditionally marginalized particularly gender continues to be under-analyzed. The bulk of research recognized the digital inclusion of girls and women as important but do not go into great detail on the underlying causes or impacts. We find that most gender-specific literature on digital inclusion is on developed economies in the northern hemisphere. We also observed a lack of user and geographical segmentation when it comes to gender, the location, and socio-economic context of the digitally excluded. In conclusion, there is an urgent need for a more in-depth analysis of the drivers of gender-specific digital inclusion and barriers resulting in exclusion, particularly so in emerging economies.
\end{abstract}

\section{CCS CONCEPTS}

- Applied computing; • Computers in other domains; • Computing in government; • E-government;

Permission to make digital or hard copies of all or part of this work for personal or classroom use is granted without fee provided that copies are not made or distributed for profit or commercial advantage and that copies bear this notice and the full citation on the first page. Copyrights for components of this work owned by others than ACM must be honored. Abstracting with credit is permitted. To copy otherwise, or republish to post on servers or to redistribute to lists, requires prior specific permission and/or a fee. Request permissions from permissions@acm.org.

ICEGOV 2021, October 06-08, 2021, Athens, Greece

(C) 2021 Association for Computing Machinery.

ACM ISBN 978-1-4503-9011-8/21/10 . \$15.00

https://doi.org/10.1145/3494193.3494211

\section{KEYWORDS}

Digital inclusion, Digital gender divide, Digital transformation, Gender, Indicators, Monitoring and measurement

\section{ACM Reference Format:}

Morten Meyerhoff Nielsen and Mercy Erhi Makpor. 2021. Digital inclusion and gender-associated indicators: A critical review of post-2010 literature. In 14th International Conference on Theory and Practice of Electronic Governance (ICEGOV 2021), October 06-08, 2021, Athens, Greece. ACM, New York, NY, USA, 6 pages. https://doi.org/10.1145/3494193.3494211

\section{INTRODUCTION}

Shaped by the digital divide and other forms of structural inequalities, the impact of the digital transformation has a significant longterm effect that are both positive and negative socio-economic consequences for marginalized persons and underrepresented communities. Estimates in 2018 show that digital devices and the internet are utilized by 327 million fewer women than men [27, 28]. The under-representation of women in key industries, management, and decision-making positions is amplified in the increasingly important ICT sector, with men being four times more likely than women to be ICT professionals. Over time this will be further entrenched as a mere $0.5 \%$ of 15 -year old girls currently aim to work in the ICT sector, compared to $5 \%$ of boys of similar age [27]. More so, containing the COVID-19 global pandemic and economic downturn have constrained the effective access and use thus cementing existing patterns of digital inclusion especially by girls.

With the Sustainable Development Goals (SDG) aiming to achieve gender equity by 2030, SDG Goal 5 - "achieve gender equality and empower all women and girls”, is specifically designed to help drive gender equality, including digital inclusion and leaving no one behind. In response to the call put out by the United Nations in The Age of Digital Interdependence [34] and the SDGs, the objective of our analysis is to take a critical look at existing academic literature, between 2020 and 2010 and the focus of the current discourse of digital inclusion relating to gender. In doing so we ask one key question: What are some of the underlying indicators used for monitoring and measuring digital inclusion and the digital gender divide identified in the academic literature to date?

Studies have shown that digital inclusion is not just about bridging the digital divide, it is also about promoting the call for the reduction of the digital gender gap that has been underway for decades [20]. [17, 18], emphasizes that 200 million fewer women than men are online, this had risen to 327 million by 2018 [28]. This implies that fewer women access online goods and services than men. Significantly, women's slower take-up of digital technology is partly the result of a masculine undertone of online content, services, and user interphases [15]. Thus, women are comparatively 
slower in acquiring advanced digital technology skills that could earn them more opportunities for online services [35]. This challenge amplifies existing factors behind the digital gender divide and gender inequality. Studies have also shown that there is a striking disparity between men and women in taking up jobs especially in technology [20], leading a majority of women to be left out of the technology revolution [4].

This has raised concerns amongst researchers, academia, and policymakers in terms of having the right data and evidence to back up plans towards involving more women and improving their technological skills. While there are consistent arguments that digital technology is indeed a concrete tool for women to overcome inequalities, digital inclusion can help bridge the digital gender gap [27]. This is achievable through engaging more girls and women in the technology, health, education, and service sectors [27, 17], however, in the absence of indicators differentiated by gender, it is difficult to measure the impact. More so, with indicators such as age, income level, literacy level, it difficult to ascertain the level of digital inclusion - or exclusion - over time $[4,13]$.

It is against this backdrop, that our analysis of the current state of affairs on digital inclusion and the digital gender divide should be seen. To explore the research question, this article is structured as follows: Section 2 outlines the methodology used in the study while section 3 summarizes the discussion of digital inclusion and digital gender divide in the literature review. Section 4 attempts to answer the research question by presenting the thematic dimensions identified in the existing literature and section 5 concludes with a set of policy recommendations and suggestions for future research.

\section{METHODOLOGY}

To explore our research question, we apply a systematic review of academic literature [12] related to the digital inclusion of women and girls. First, relevant publications were identified and retrieved from the Scopus repository. Scopus was chosen as it is the most comprehensive global repository of English language academic literature. The search was complemented by similar searches in Mendeley and Google Scholar to ensure as complete a sample as possible. Secondly, dimensions to structure the review were defined. These keyword dimensions were identified together with the indicators used in various digital inclusion publications specific to the traditionally marginalized sub-groups of women including age, education, ethnicity, geographical location, etc.

For the first (step 1), we carried out a keyword query (in SCOPUS?) using "digital inclusion" in the "All social sciences" category. The "All social sciences" category covers "Arts and Humanities (ARTS)", Business, Management and Accounting (BUSI)" "Decision Sciences (DECI)", "Economics, Econometrics and Finance (ECON)", "Psychology (PSYC)" and "Social sciences (SOCI)". The query also searched for results containing concepts such as "digital gender divide", "digital divide", "digital transformation" and "systemic review". We did not screen for terms such as data, indicators, statistics, measurement, or monitors in the literature. The aim of excluding these terms was to identify both the themes or causes within the literature and what data and indicators have been used, or suggested directly or indirectly. An initial search was carried out in December
2020 with a total number of 8,527 publications from 1st January 1951 to 1 st December 2020.

In the second (step 2), we refined the result by limiting the search period from 1st January 2010 to 1 st December 2020. Significantly, the internet emerged in the mid-1990s; however, its wide global adoption started in both developed and developing economies in the last decade, thus we chose 2010 as a cut-off point. Key observations and conclusions made prior to 2010 regarding the digital gender divide and digital inclusion, are expected to be reflected in our post-2010 sample. Limiting our search to literature published after 1st January 2010, resulted in 6,271 publications.

Third (step 3), we applied an inward search which resulted in 208 publications. Keywords such as "men' and "women”, "male” and "female", "boys" and "girls". The keywords were used to identify the key academic publications for our analysis. The result falls into two broad topical clusters. The first resulting in 116 publications (56\%) focused on digital technology and e-health solutions in healthcare provision, assisted living, and home care for adults and the elderly. While interesting, this set of publications do not fall within the scope of our research question and the focus on digital inclusion and the digital gender divide per se. The second cluster consists of 92 publications (44\%) focused mainly on both digital inclusion and the digital gender divide. However, intertwining both academic concepts are digital technology access and affordability, skills and usage which acted as either precondition causes or facilitators. These were a considerable match for the focus of our article and research question, thus constituting our final sample size.

\section{WHAT LITERATURE TELLS US}

The analytical focus of the 92 publications identified, vary, as expected, depending on the publication in question, not least in relation to various user groups and segments assessed - both in general terms and in terms of gender. A number of common sub-themes nonetheless emerge across the 92 publications during the screening for indicators used or proposed for monitoring and measurement of digital inclusion and the digital gender divide. To identify the core themes, we mapped the publications in relation to their primary and secondary focus. Within the broader topic of the digital gender divide, the dominant thematic cluster $(n=44,47 \%)$ is the genderbased digital exclusion as part of a wider deliberation of digital inclusion. The first thematic cluster highlighted focus on the underlying causes of the digital gender gap. Dominating gaps are issues related to nature of gender itself and gender roles, digital gender divide and gender-based digital exclusion. While rarely quantified, authors do suggest a number of ways to improve digital inclusion process especially in rural communities $[2,26]$. These include roll out of high-quality internet access, tailored digital skills development and free online education targeting rural areas. The second thematic cluster identified is financial inclusion $(n=20,22 \%)$ and how technology can eliminate the digital gender gap by creating innovative solutions to ease access to financial services such as mobile money, money transfers, credit, and micro-loans [1, 14, 23]. Here examples such as mobile money, the facilitation of efficient and effective financial service have been highlighted as good practice examples to potentially be emulated $[14,22,23]$. The third thematic 
Table 1: Major areas covered in screened population (Source: Authors)

\begin{tabular}{lll}
\hline Main thematic issues covered & Number of publications & $\%$ Rate \\
\hline The gender gap, the digital gender divide, and gender-based digital exclusion & 44 & 47 \\
Financial inclusion & 20 & 22 \\
ICT enabled healthcare and assisted living & 9 & 10 \\
Digital communication & 2 & 2 \\
Culture context and diversity & 11 & 12 \\
Miscellaneous & 6 & 7 \\
Total & 92 & 100 \\
\hline
\end{tabular}

cluster of publications focused on technology in healthcare provision and assisted living $(n=9,10 \%)$. Authors particularly highlighted the challenges related to infrastructure and how to gain access to information on health care and how these issues impact women. Essentially the focus is on gender inequalities resulting from a lack of equal, or unequal access to health care services especially in rural regions and how the digitization of information and service offers cement such patters further [6,7]. Key proposals to overcome these challenges include technological support for women in rural regions and capacity development to build skills for women to be able to use digital health applications and services when the need arises. A fourth cluster $(n=2,2 \%)$ focused on digital communication as a tool to enable access to services, decision-makers, or jobs opportunities. Proposals on how to address this includes [7, 21]. A fifth thematic cluster focus on digital inclusion with respect to race, culture, and diversity $(n=11,12 \%)$. Linguistic heterogeneity, race, culture, ethnicity, diversity, and religion considerations dominate the literature in this cluster. This is particularly seen in relation to active participation in decision making, social discourse and citizenship, but also how it may bring about demographic challenges in countries at both local and national levels [21, 22]. The final group $(n=6,7 \%)$ covered a multitude of issues related to digital inclusion and the digital gender gap in general terms, such as social inequalities arising from unequal access to digital technology. Much of the research in this cluster also touch upon issues such as gender, region, race, ethnicity, social class, etc. Some researchers have suggested inclusion, tackling stereotypes, raising awareness as well as setting policy actions as ways to address such negative impacts. This facilitated the mapping of key themes discussed within the literature but also to identify the indicators use, or proposed directly or indirectly in the past. Table 1 summarizes the main thematic clusters identified all the 92 publications on digital inclusion, the digital gender divide, and other gender inequalities.

Generally, all sample publications address topics around women, girls, education and technology, especially in rural areas. This is highlighted in relation to both the various kinds of inequalities and exclusion, especially the lack of access to and affordability of internet access and digital devices, and the lack of traditional but especially digital literacy.

With respect to SDG 5, women in rural areas still disproportionately lag behind in digital technology - and how it may potentially be addressed $[15,19]$. In the analysis, all 92 publications indicate the importance of three indicators. That is, access and affordability as required precondition causes for digital inclusion, the lack of skills as a cause of digital exclusion or facilitator of digital inclusion, and the use of digital content and solutions as an indicator of outcomes.

To explore our research question, we will therefore review the literature in relation to digital inclusion as such, the digital gender divide, accessibility, skills, and usage.

\subsection{Digital Inclusion}

Analyzing the sample literature, it becomes clear that the term "digital inclusion" goes beyond mere access to technology devices and the internet. Observation of the term holds across the academic research, policy frameworks, and practical executions. Over time, there has been a move towards a deeper understanding of the concept and the skills and capacities needed to ensure its effective achievement.

Currently, there is no universally accepted definition of the term as it covers almost every imaginable aspect within and across various academic domains $[14,29,30]$. As a result, the term digital inclusion is often adapted to reflect the perspective of a given author. As an all-in-one phrase, "digital inclusion" is regularly used to refer to digital, financial, social, economic or political, inclusion [29]. Reference to the concept is made concerning equality and equity in areas such as education, health care, jobs, public and private services, as well as decision-making processes [19]. It was also observed that the single term "inclusion", is often associated with a sense of justice, and compassion [6], and more so viewed as a panacea for the promotion of equality especially amongst the marginalized.

The analysis finds that digital inclusion is in the literature associated with diversity and technology, supporting earlier observations made by e.g. [29] and [33]. The initial reference stems from the concepts of the digital divide and digital literacy and how some individuals do not have access to digital technology as highlighted by [15] and [19].

Based on the literature review, a common definition of digital inclusion would be "the ability to access and use digital technology to create economic and personal value". This definition is closely associated with those suggested [19] and [4] respectively. The description implies that the digital inclusion of marginalized groups is characterized by geographical location, population density, age, gender, formal and informal educational attainment levels, occupation type, and relative household income. Any analytical matrix used to assess digital inclusion must, therefore, go beyond simple access to the internet and technology devices. Thus, the digitally marginalized groups should be seen as individuals within a given 
context who are subjected to inequalities through a lack of access to digital infrastructure and digital technology tools as a result of location, age, gender, skills, and/or affordability [27, 28].

\subsection{Digital gender divide}

The literature review finds that the "digital divide" as a term is applied in similar ways as digital inclusion. That is that no agreed definition exists and that authors tend to adjust it to their specific research focus. By comparison, the "digital gender divide", stems from a collection of social and academic perspectives on the digital transformation and inclusion in this process [24, 25]. Some scholars see this as problematic from an ideological perspective, as it may result in a technologically deterministic approach and bias [29, 33], and in turn result in a lack of distinctive philosophies on the socioeconomic settings within which the marginalized groups live $[11,23]$.

Furthermore, the socio-economic settings associated with the digital gender divide are structured within certain key demographic variables and social inequalities as emphasized by [27]. Examples are socio-economic status, age, gender, ethnicity, geographic location, etc. According to the literature, the digital gender divide thus becomes a convenient catch-all metaphor to explain some of the social inequalities synonymous with the digital transformation of society $[19,23,24]$.

To date, academic research on the digital gender divide has largely been confined to global and national parameters. Academics see the digital gender divide as springing from a limitation of technical skills, the limited physical access to technology as well as social inequalities [18, 25, 29]. Researchers like [9] have highlighted that a more thorough understanding of inequalities requires an understanding of the theoretical context of digital technology and its influence on social inequalities, emphasizing the importance of exploring how access to digital technology drives or reinforces inequalities and digital divides, such as the prospect for economic mobility, social engagement and inclusion [36]. Although the statistical evidence is limited this is supported by early empirical evidence which in 2011 estimated that four out of every five women live in developing economies experience gender-specific discrimination [13].

Like research on digital inclusion in general, data used tend to focus on internet access and traditional skills (i.e. reading, writing, mathematics) from sources like the ITU, UNESCO, the World Bank, etc $[8,30,32]$. While relevant this does not specifically monitor or measure the digital gender divide, nor does it identify the geographical location of the potentially excluded, or any socio-economic indicators such as age, income, or educational attainment levels. Such indicators and segmentation are key for the basic assessment, monitoring, and measurement of the level of the digital gender divide.

\subsection{Access and Affordability}

The quality of access, access to electricity, the internet, technology devices, and other technical infrastructures were seen as interconnected elements that acted as preconditional causes of digital inclusion even though these are significant aspects of digital inclusion. Similarly, emphasis on the role of digitization in enhancing the financial inclusion of women $(n=20$ or $22 \%)$ was a key theme in the literature $[2,4,37]$. Both accessibility and affordability act as indicators which are also regarded as having the ability to fully take part in a digital society $[10,18]$. However, women worldwide are affected by either the lack of affordability or limitations in access to digital technology [15]. These were also observed to be interconnected to the thematic causes listed in table 1 above. Thus, while people globally are increasingly online, statistics show that the relative number of women without access to the internet has risen from $11 \%$ in 2013 to $13 \%$ globally at the end of 2016 . That is, 250 million fewer women have access to online content and can afford online public services compared to men in 2016 [16].

This trend has also been observed by the OECD that 327 million fewer women than men had access to digital technology and the internet globally between the periods of 2013 and 2017 [28]. Over half of the identified publications $(n=44$ or $47 \%$ ) emphasized that the digital gender gap is widening and does so at an increasing rate. Accordingly, women globally are on average $26 \%$ less likely than men to afford digital devices such as smartphones, computers, and other digital technology devices. In places such as Africa and South Asia, these proportions stand at approximately $70 \%$ and $34 \%$, respectively [28]. Publications which focused on eHealth and ICTenable assisted living ( $\mathrm{n}=9$ or $10 \%$ ) emphasized on women's lack of access and

Data used tend to focus on internet access, the type of access (e.g. fixed or mobile connectivity), access to electricity, and the like [28, $36,37]$. Data is usually sourced from international organizations such as ITU and UNESCO, where affordability is often found in sources from the private sector or NGOs [14, 15, 33]. While relevant this does not specifically monitor or measure the digital gender divide, nor does it identify the geographical location of the potentially excluded, or segment indicators in relation to age, income, or educational attainment levels. Such indicators and segmentation are essential to make a basic assessment of digital inclusion and the digital gender divide.

\subsection{Skills}

Skills are seen to include both traditional and digital competencies, as well as critical thinking, and literacy [24]. Our in-depth review of all 92 publications finds that not only does lack of digital skills remains one of the major barriers to the digital gender divide [16] but also poses a critical challenge to some of the themes highlighted in table 1 above. Digital skills are essential in light of the digital transformation as new technologies and employment opportunities require new and increased levels of digital skill, not least concerning the theme of digital communication $(n=2,2 \%)$ identified in the literature review. Not all women skillfully make use of digital technology, hence, the vast difference in the digital gender divide [24, 25]. For instance, while ICT specialist skills are becoming more significant, findings indicate that the gap for digital skills and competencies by gender is set to widen especially in developing countries [15, 24]. Traditional skills set in the form of reading and writing are key indicators used in the digital inclusion and digital gender divide literature to date [10, 37]. Such data is often source from the likes of UNESCO database on skills and educational attainment levels globally $[10,14,32]$. Generally, data for 
high and medium/income countries are more readily available than from their low-income peers [24]. Often missing but suggested is the need to include indicators for digital competencies not least in developing countries [31]. Traditional skills and capacity indicators are complimented with skills such as the use of the internet, coding skills, or the level of STEM graduates as a percentage of the population [41,33]. Eurostat and European countries collect data on a set of digital skills such as the use of computers, mobile devices, internet use, various types of internet use (e.g. social media, eCommerce, eBanking, online government services), the skill of using text or numerical processing software, looking for information, using online forms, coding, etc $[8,14,15]$. Unfortunately, gender is rarely an indicator measured except for the number of female STEM graduates, or gender in relation to traditional education. Some like the Australian Digital Inclusion Index, Eurostat, and some of the Pew International surveys focus on age, and educational attainment level, and income $[8,35]$. Few International often do surveys on different race and minority indicators in a US context, thus falling within the identified literature on culture context and diversity identified in the literature review $(n=11,12 \%)$.

\subsection{Usage}

Usage as an indicator of digital content and solutions is not always measured. It is the actual use of digital technology and services which creates value. Usage acts as an indicator of places of access to measure actual value creation and digital inclusion of marginalized communities [10]. Studies show that the use of the internet continues to grow, with more than 4.1 billion people online globally [16]. However, it continues to be influenced by geography, age, educational attainment level difference, ethnicity, religion, etc. This often indicates an outcome in limitation of some of the themes mentioned in Table 1 above.

Estimates highlight that men continue to be more likely users of ICT tools and resources than women [23]. As a result, the debate and challenges continue to be focused on how fundamental a challenge irregular and insufficient use of ICT is and how important it is to close the digital gender divide $[22,25]$ where the pace of internet take-up by women in developing countries, compared to developed countries [15], is resulting in both an increasing digital divide between developed and less developed economies, as well as an increasing digital gender divide.

Indicators measuring the use of public and private digital services, digital products, and content such as eHealth services, social and civic engagement activities should therefore be considered. This would build on existing indicators of internet and mobile device use, by complementing these with the use of social media, eBanking, eCommerce, and online government services. This would allow us to triangulate different indicators to see if there are opportunities or untapped potential in promoting different types of services and usages across user segments. Such indicators are already collected by many national statistical agencies in e.g. Australia, Brazil, South Africa, the majority of European countries, or the international benchmarks such as the DESI and Eurostat. Like the observations for access, affordability, and skills specific monitor or measure digital gender divide, data is rarely available in relation to the geographical location of the users of the internet or the various types of online content and services. Similarly, any socio-economic segmentation by age, income or educational attainment levels is largely missing. Such elements are essential to make a basic assessment of the level of the digital gender divide.

\section{CONCLUSION AND RECOMMENDATION}

The study analyzed and described some of the monitoring indicators for checking the digital gender divide identified in the academic literature from 2010 to January 2021. It maps out what the post-2010 academic literature suggests as a means to help reduce or possibly eliminate the digital gender divide and improve digital inclusion of girls and women. We find that majority of the studies have been conducted within the OECD countries, especially in Europe. Some have been conducted in countries such as Canada, Japan, and the USA [27]. The literature and data on which it is based show that a large global digital gender gap persists. Studies conducted have often taken place with limited consideration of developing countries and emerging economies. According to the literature, a disturbing presence of inequalities and the lack of indicators such as access, skills usage, connectivity, and affordability do persist [11, 18]. In policy terms, we observe that digital inclusion generally combines three main concepts and proposes three potential influential indicators related to the digital inclusion of girls and women. That is: accessibility as a precondition, skills as a facilitator, and usage as an outcome.

Complementing our review of the post-2010 literature review with an analysis of the data sources used, we find that current indicators show a surprising lack of geographical and user segmentation. Not only in terms of gender, but in relation to geographical location, age, income, and educational attainment levels. Thus, resulting in a lack of statistical foundation to measure and monitor digital inclusion and the digital gender divide. There is thus an urgent need for a more in-depth understanding and analysis of the specific drivers of gender-specific digital inclusion and barriers resulting in exclusion, particularly in emerging economies, but across geographical locations, gender, age, income, and educational attainment levels. Similarly, there is need for user and geographical data segmentation in both national and international benchmarks linked to digital transformation, digital inclusion, and the SDGs so decisions makers can act on the insight provided by data. There is a need for new gender-focused inclusive policy frameworks and strategies to address the widening digital divide and gender gap observed. Failing to address the widening digital divide and particularly the digital gender gap will present risks for policymakers tasked with the goal of attaining technological governance as well as commitments to the 2030 SGDs. The study, therefore, proposes the need for further research on the availability of relevant indicators to advance gender-inclusive policy approaches and initiatives to successfully curb the current digital gap and impact positively on the traditionally marginalized.

\section{ACKNOWLEDGMENTS}

This article partially based on research carried out by the authors in connection with the "Measuring at the margins: A global framework for digital inclusion" Project for the Digital Future Society. The article is the result of the project "SmartEGOV: Harnessing EGOV for 
Smart Governance (Foundations, methods, Tools)/NORTE-01-0145FEDER-000037”, supported by Norte Portugal Regional Operational Programme (NORTE 2020), under the PORTUGAL 2020 Partnership Agreement, through the European Regional Development Fund (EFDR). It was also supported in part by the European Commission (OpenGovIntelligence H2020 grant 693849), and Estonian Research Council (PUT773, PUT1361).

\section{REFERENCES}

[1] Aricat, R. G. (2015). Is (the study of) mobile phones old wine in a new bottle? A polemic on communication-based acculturation research. Information Technology \& People.

[2] Akanbi, B. E., \& Akanbi, C. O. (2012). Bridging the digital divide and the impact on poverty in Nigeria. Computing, Information Systems \& Development Informatics, 3(4), 81-87.

[3] Albino, V., Berardi, U., \& Dangelico, R. M. (2015). Smart cities: Definitions, dimensions, performance, and initiatives. Journal of urban technology, 22(1), 3-21.

[4] Al-Jaghoub, S., \& Westrup, C. (2009). Reassessing social inclusion and digital divides. Journal of Information, Communication, and Ethics in Society, 7(2/3), 146-158.

[5] Alvaredo, F., Chancel, L., Piketty, T., Saez, E., \& Zucman, G. (Eds.). (2018). World inequality report 2018. Belknap Press.

[6] Antonio, A., \& Tuffley, D. (2014). The gender digital divide in developing countries. Future Internet, 6(4), 673-687.

[7] Cimpoieru, C. (2011, June). Digital inclusion of the elderly: an ethnographic pilot-research in Romania. In International Conference on Digital Information and Communication Technology and Its Applications (pp. 663-677). Springer, Berlin, Heidelberg.

[8] Davis, R., Garder, J., \& Schnall, R. (2020). A review of usability evaluation methods and their use for testing eHealth HIV interventions. Current HIV/AIDS reports, 17(3), 203.

[9] DeGuzman, P.B., Bernacchi, V., Cupp, C. A., Dunn, B., Ghamandi, B. J., Hinton, I D.,. . .\& Sheffield, C. (2020). Beyond broadband: digital inclusion as a driver of inequalities in access to rural cancer care. Journal of Cancer Survivorship, 14(5), 643-652.

[10] Digital Future Society. (2019). Measuring the margins: A global framework for digital inclusion. Available at https://digitalfuturesociety.com/reports/ Accessed on the 12th March 2021

[11] Gilbert, M. (2010). Theorizing digital and urban inequalities: Critical geographies of 'race', gender and technological capital. Information, communication \& society, 13(7), 1000-1018.

[12] Hansen, H., \& Trifkovic, N. (2013). Systematic reviews: Questions, methods and usage.

[13] Heeley, M., \& Damodaran, L. (2009). Digital inclusion: A review of international policy and practice. Loughborough University.

[14] Helsper, E. (2008). Digital inclusion: an analysis of social disadvantage and the information society. Department for Communities and Local Government.

[15] Hilbert, M. (2011, November). Digital gender divide or technologically empowered women in developing countries? A typical case of lies, damned lies, and statistics. In Women's Studies International Forum (Vol. 34, No. 6, pp. 479-489). Pergamon

[16] International Telecommunication Union News Magazine. (2016). How can we close the digital gender gap? Available at: www.itu.int/en/itunews/Documents/ 2016-04/2016_ITUNews04-en.pdf. Accessed on the 14th of February, 2021.

[17] International Telecommunication Union Press Release. (2019). New ITU data reveal growing internet uptake but a widening digital gender divide. Available at www.itu.int/en/mediacentre/Pages/2019-PR19.aspx. Accessed on 7th February 2021.

[18] International Telecommunication Union. (2020). Affordability. Available at: www.itu.int/en/mediacentre/backgrounders/Pages/affordability.aspx. Accessed on 23rd March 2021

[19] Jaeger, P. T., Bertot, J. C., Thompson, K. M., Katz, S. M., \& DeCoster, E. J. (2012) The intersection of public policy and public access: Digital divides, digital literacy, digital inclusion, and public libraries. Public Library Quarterly, 31(1), 1-20.

[20] Kularski, C., \& Moller, S. (2012). The digital divide as a continuation of traditional systems of inequality. Sociology, 5151, 1-23.
[21] Lawrence, S., \& Davis, C. (2019). Fans for diversity? A critical race theory analysis of Black, Asian and Minority Ethnic (BAME) supporters' experiences of football fandom. International fournal of Sport Policy and Politics, 11(4), 701-713.

[22] Lundedal Hammar, E. (2015). Ethical recognition of marginalized groups in digital games culture. Proceedings of Digital Games Research Association (DiGRA)

[23] Manda, M. I., \& Backhouse, J. (2017). Digital transformation for inclusive growth in South Africa. Challenges and opportunities in the 4th industrial revolution. In 2nd African Conference on Information Science and Technology, Cape Town, South Africa.

[24] Martínez-Cantos, J. L. (2017). Digital skills gaps: A pending subject for gender digital inclusion in the European Union. European Journal of Communication, 32(5), 419-438.

[25] Min, S. J. (2010). From the digital divide to the democratic divide: Internet skills, political interest, and the second-level digital divide in political internet use. Journal of Information Technology \& Politics, 7(1), 22-35.

[26] Natile, S. (2019). Regulating exclusions? Gender development and the limits of inclusionary financial platforms. International Journal of Law in Context, 15(4), 461-478.

[27] Organization for Economic Cooperation and Development. (2017). OECD Digital economic outlook, 2017. Available at www.skillsforemployment.org/edmsp1/ groups/skills/documents/skpcontent/ddrf/mtkz/ edisp/wcmstest4_193707.pdf. Accessed on the 6th of March 2021.

[28] Organization for Economic Cooperation and Development. (2018). Achieving inclusive growth in the face of digital transformation and the future of work. Available at www.oecd.org/g20/OECD Achieving\%20inclusive\% 20 growth $\% 20$ in $\% 20$ the $\% 20$ face $\% 20$ of $\% 20$ FoW.pdf. Accessed on the 20 th February 2021.

[29] Organization for Economic Cooperation and Development. (2018). Bridging the digital gender divide: Include, upskill, innovate. Available at www.oecd.org/internet/bridging-the-digital-gender-divide.pdf. Accessed on 23rd January 2021.

[30] Ragnedda, M., \& Muschert, G. W. (Eds.). (2013). The digital divide: The Internet and social inequality in the international perspective. Routledge.

[31] Ramírez-Plascencia, D., \& Alonzo-González, R. M. (2020). Ending the Digital Gender Divide. Are Coding Clubs the Solution?. Tripodos, (46), 29-41.

[32] Thompson, K. M., Jaeger, P. T., Taylor, N. G., Subramaniam, M., \& Bertot, J. C. (2014). Digital literacy and digital inclusion: Information policy and the public library. Rowman \& Littlefield.

[33] Tsatsou, P. (2011). Digital divides revisited: what is new about divides and their research?. Media, Culture \& Society, 33(2), 317-331.

[34] United Nations Secretary-General's High-level Panel on Digital Cooperation. (2019). The age of digital interdependence. Available at www.un.org/en/pdfs/ DigitalCooperation-\%20report-for\%20web.pdf. Accessed on 20th January 2021.

[35] Van Dijk, J. A. G. M. (2012). The evolution of the digital divide: The digital divide turns to inequality of skills and usage. Digital enlightenment yearbook, 2012, 57-75.

[36] World Benchmarking Alliance. (2019): Available at https://parispeaceforum.org/ place/the-world-benchmarking-alliance/. Accessed on the 28th March 2021

[37] World Economic Forum. (2018). Women are still on the wrong side of the digital skills gap. Available at www.weforum.org/agenda/2018/07/as-technologyadvances-women-are-left-behind-in-digital-divide. Accessed on 19th March 2021.

[38] United Nations Educational, Scientific and Cultural Organization. (2019). Skills for work and life. Available at Skills for work and life (unesco.org). Accessed on $29^{\text {th }}$ April 2021.

[39] Global System for Mobile Communications. (2019). Mobile connectivity index: Methodology. Available at GSMA-Mobile-Connectivity-Index-Methodology2019.pdf. Accessed on $29^{\text {th }}$ April 2021.

[40] European Union Science Hub. (2019). The digital competence framework 2.0. Available at The Digital Competence Framework 2.0 | EU Science Hub (europa.eu). Accessed on $29^{\text {th }}$ April 2021.

[41] Thomas, J., Barraket, J., Wilson, C. K., Rennie, E., Ewing, S., \& MacDonald, T. (2019). Measuring Australia's Digital Divide: The Australian Digital Inclusion Index 2019, RMIT University and Swinburne University of Technology, Melbourne, for Telstra.

[42] United Nations News. (2021). Don't let the digital divide become 'the new face of inequality': UN deputy chief. Available at Don't let the digital divide become 'the new face of inequality': UN deputy chief || UN News. Accessed on $29^{\text {th }}$ April 2021. 